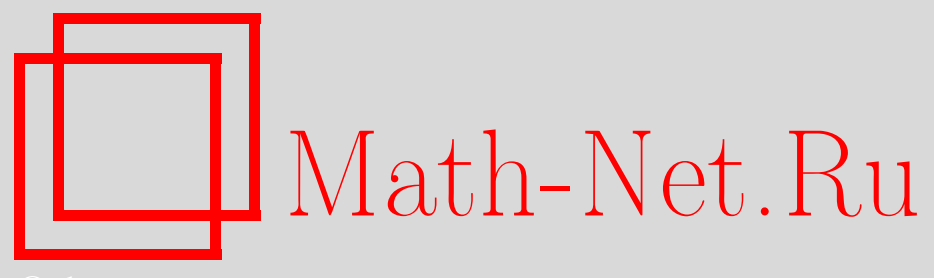

С. В. Тихонов, Об отсутствии кратного перемешивания и централизаторе сохраняющих меру действий, Матем. заметки, 2015, том 97, выпуск 4, 636-640

DOI: https://doi.org/10.4213/mzm10541

Использование Общероссийского математического портала Math-Net.Ru подразумевает, что вы прочитали и согласны с пользовательским соглашением http://www . mathnet.ru/rus/agreement

Параметры загрузки:

IP : 54.162 .127 .20

26 апреля 2023 г., 16:15:37

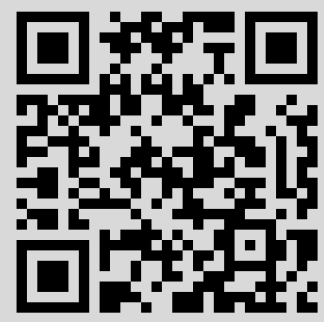




\section{Об отсутствии кратного перемешивания и централизаторе сохраняющих меру действий}

\section{С. В. Тихонов}

Преобразованиями в работе называются обратимые сохраняющие меру отображения единичного отрезка в себя. Такие преобразования являются основным предметом изучения абстрактной теории динамических систем.

Непрерывным действием топологической группы $G$ называется набор преобразований $T=\left\{T^{g}\right\}_{g \in G}$, удовлетворяющий следующим двум условиям:

1) $T^{h} T^{g}=T^{h g}$ для всех $g, h \in G$;

2) для любых измеримых множеств $A$ и $B$ отображение $g \mapsto \mu\left(T^{g} A \cap B\right)$ непрерывно.

Последовательность преобразований $\left\{T_{i}\right\}$ называется перемешивающей, если для любых измеримых множеств $A, B$ имеем

$$
\mu\left(T_{i} A \cap B\right) \rightarrow \mu(A) \mu(B)
$$

при $i \rightarrow \infty$.

Преобразование (или действие) $T$ называется перемешивающим, если последовательность $\left\{T^{g_{i}}\right\}$ перемешивает, как только $g_{i}$ стремится к бесконечности. Последнее означает, что последовательность $\left\{g_{i}\right\}$ имеет только конечное число членов в любом компакте.

Аналогично, перемешивающее действие называется двукратно перемешивающим, если для любых перемешивающих последовательностей $\left\{T_{i}\right\},\left\{S_{i}\right\},\left\{T_{i}^{-1} S_{i}\right\}$ его элементов и любых множеств $A, B, C$ имеем

$$
\mu\left(T_{i} A \cap S_{i} B \cap C\right) \rightarrow \mu(A) \mu(B) \mu(C)
$$

при $i \rightarrow \infty$.

В дальнейшем все рассматриваемые последовательности $\left\{T_{i}\right\},\left\{S_{i}\right\},\left\{T_{i}^{-1} S_{i}\right\}$ предполагаются перемешивающими.

Свойство двукратного перемешивания введено Рохлиным. В работе [1] он указал один из классов преобразований, для которого двукратное перемешивание следует из обычного перемешивания.

На данный момент найдены и другие классы преобразований (и действий), обладающие таким свойством. Обзор результатов на эту тему, полученных разными авторами, можно найти в [2].

До сих пор неизвестно, существуют ли перемешивающие преобразования, не перемешивающие двукратно. Этот вопрос называется проблемой Рохлина о кратном перемешивании.

О действиях групп известно больше. Для некоторых групп из перемешивания следует двукратное перемешивание, для других групп это не так. В частности, для групп $\mathbb{Z}^{d}$ при $d>1$ имеются контрпримеры.

Все они в том или ином смысле основаны на рассуждениях Ледрапье [3].

УтверждениЕ 1 (Трехточечный пример Ледрапье). Существует перемешивающее действие $L$ двумерной решетки $\mathbb{Z}^{2}$ и множество $A, \mu(A)=1 / 2$, такие, что

$$
\mu\left(L^{\left(2^{k}, 0\right)} A \cap L^{\left(0,2^{k}\right)} A \cap A\right)=0
$$

для любого натурального $k$.

Таким образом, L перемешивает, но не перемешивает дважды.

DOI: $10.4213 / \mathrm{mzm} 10541$

C) С. В. Тихонов, 2015 
Отметим, что согласно результатам [4], [5] типичное перемешивающее действие группы $\mathbb{Z}^{d}$ перемешивает дважды, т.е. пример Ледрапье является "исключением из правил".

В настоящей работе изучаются действия произвольной топологической абелевой группы, имеющие тот же предел, что и в примере Ледрапье. Дадим соответствующее определение.

ОПРЕДЕЛЕниЕ 1 . Действие топологической группы имеет $L$-nредел, если существуют последовательности ${ }^{1}\left\{T_{i}\right\},\left\{S_{i}\right\}$ и множество $A, \mu(A)=1 / 2$, такие, что

$$
\mu\left(T_{i} A \cap S_{i} A \cap A\right) \rightarrow 0 .
$$

Действия, обладающие $L$-пределом, назовем $L$-системами. В частности, $L$-системой является упомянутый выше пример Ледрапье.

УтвеРЖДЕнИЕ 2. Пусть задана произвольная L-система и преобразование $U$, коммутирующее с элементами последовательностей $\left\{T_{i}\right\},\left\{S_{i}\right\},\left\{T_{i}^{-1} S_{i}\right\}$. Тогда мера $\mu(U A \cap A)$ равна либо $1 / 2$ либо $1 / 4$.

ДокАзАтельство. Мы воспользуемся тем, что при $i$, стремящемся к бесконечности, имеют место сходимости

$$
\begin{gathered}
T_{i} A \rightarrow\left(S_{i} A \triangle A\right)=\left(S_{i} A \cap \bar{A}\right) \cup\left(S_{i} \bar{A} \cap A\right), \\
S_{i} A \cap A \rightarrow T_{i} \bar{A} \cap A .
\end{gathered}
$$

Обозначим меру $\mu(U A \cap A)$ через $x$. Вычисление $x$ основано на оценке различными способами выражений $\mu\left(U T_{i} A \cap S_{i} A \cap A\right)$.

Зафиксируем произвольное $\varepsilon>0$ и будем использовать обозначение $a \stackrel{\varepsilon}{\sim} b$, если модуль разности между числами $a$ и $b$ меньше $\varepsilon$.

Тогда при достаточно большом $i$ по формуле (3) имеем

$$
\begin{aligned}
\mu\left(U T_{i} A \cap S_{i} A \cap A\right) & \stackrel{\varepsilon}{\sim} \mu\left(U S_{i} A \cap U \bar{A} \cap S_{i} A \cap A\right)+\mu\left(U S_{i} \bar{A} \cap U A \cap S_{i} A \cap A\right) \\
& =\mu\left(S_{i}(U A \cap A) \cap(U \bar{A} \cap A)\right)+\mu\left(S_{i}(U \bar{A} \cap A) \cap(U A \cap A)\right) \\
& \stackrel{2 \varepsilon}{\sim} 2 \mu(U A \cap A) \mu(U \bar{A} \cap A)=x(1-2 x) .
\end{aligned}
$$

С другой стороны, применяя формулу (4), имеем

$$
\mu\left(T_{i} U A \cap S_{i} A \cap A\right) \stackrel{\varepsilon}{\sim} \mu\left(T_{i} U A \cap T_{i} \bar{A} \cap A\right) \stackrel{\varepsilon}{\sim} \mu(U A \cap \bar{A}) \mu(A)=\frac{1}{2}\left(\frac{1}{2}-x\right) .
$$

Таким образом,

$$
\frac{1}{2}\left(\frac{1}{2}-x\right) \stackrel{5 \varepsilon}{\sim} 2 x\left(\frac{1}{2}-x\right) .
$$

В силу произвольности $\varepsilon$ заключаем, что

$$
\frac{1}{2}\left(\frac{1}{2}-x\right)=2 x\left(\frac{1}{2}-x\right)
$$

Значит, есть только два варианта: $x=1 / 2$ и $x=1 / 4$.

СледствиЕ 1. Любое перемешивающее преобразование, коммутирующее с L-cистемой, имеет лебеговскую компоненту в спектре.

\footnotetext{
${ }^{1}$ Напомним, что эти последовательности предполагаются перемешивающими.
} 
ДоказАтельство. Пусть $V$ - рассматриваемое преобразование. Взяв в качестве $U$ любое множество из набора $\left\{V^{k} A\right\}_{k \in \mathbb{Z}}$, получаем, что

$$
\mu\left(V^{k} A \cap A\right)=\frac{1}{4}
$$

что является достаточным условием для наличия лебеговской компоненты.

ЗАмЕчАНИЕ 1. Это следствие связано с несколькими известными результатами в эргодической теории. Перемешивающее, но не перемешивающее дважды действие имеет нетривиальное самоприсоединение. Согласно работе Рыжикова [6] любое перемешивающее преобразование из его централизатора обладает тем же самоприсоединением. Результаты статьи Оста [7] гарантируют, что это преобразование имеет абсолютно непрерывную компоненту в спектре. Мы доказываем, что имеется лебеговская компонента, но для более узкого класса действий - для $L$-систем.

К. Шмидт доказал, что централизатор примера Ледрапье тривиален (сообщение на конференции в Варвике). Аналог этого результата для $L$-систем не верен. В частности, квадрат примера Ледрапье имеет нетривиальный централизатор.

Утверждение 2 имеет ряд следствий для действий различных групп.

СледствиЕ 2. Потоки (т.е. действия группы $\mathbb{R}$ ) не обладают L-пределом.

ДокАЗАТЕЛЬСтво. Предположим противное, и у нас есть поток $L$ с таким пределом. Так как $\mu\left(L^{\alpha} A \cap A\right)$ - непрерывная функция от $\alpha$, принимающая значение $1 / 2$ в нуле и стремящаяся к $1 / 4$ при $\alpha \rightarrow \infty$, то можно выбрать такое $\alpha$, что

$$
\mu\left(L^{\alpha} A \cap A\right)=\frac{3}{8},
$$

что противоречит утверждению 2.

Следствие 2 имеет естественное обобщение.

СледствиЕ 3. Пусть некоторое перемешивающее действие произвольной группы коммутирует со слабо перемешивающим потоком $P$. Тогда оно не обладает $L$-пределом.

ДокАзАтельство. Как и в предыдущем случае, для любого множества $A, \mu(A)=1 / 2$, в потоке $P$ найдется элемент $U$ такой, что

$$
\mu(U A \cap A)=\frac{3}{8}
$$

значит, по утверждению 2 не существует $L$-предела с множеством $A$.

СледствиЕ 4. Многомерные потоки (т.е. действия группы $\mathbb{R}^{d}$ ) не имеют L-предела.

Действительно, в централизаторе каждого такого действия имелся бы (одномерный) поток, что противоречит предыдущему следствию.

СлЕДСтвиЕ 5. Действие из утверждения 1 не вкладывается в действие плоскости.

ДокАзАтЕЛЬство. Такое действие имело бы $L$-предел, что противоречит предыдущему следствию. 
Обобщения. Некоторая модификация рассуждений утверждения 2 позволяет доказать следующий факт.

УтвеРждение 3. Пусть действие произволъной группы обладает L-пределом. Пусть также преобразования $U$ и $V$ коммутируют с последовательностями $\left\{T_{i}\right\},\left\{S_{i}\right\},\left\{T_{i}^{-1} S_{i}\right\}$, а множества $U A, V A$ и $A$ различны.

Тогда мера

$$
\mu(U A \cap V A \cap A)
$$

может принимать только значения 0 или $1 / 8$.

ДокАЗАтЕльство. Это утверждение является обобщением теоремы 1.1 из [8].

СледСтвиє 6. Преобразование не может иметь L-предел.

ДокАЗАтЕльство. Предположим, что преобразование $Q$ имеет $L$-предел. Тогда для некоторых $i, j$ имеем

$$
A=Q^{i} A \triangle Q^{j} A .
$$

Для перемешивающего преобразования это невозможно, см., например, [9].

Следствие 7. Для перемешивающего действия группы $\mathbb{Z}^{d}\left(\right.$ или $\left.\mathbb{R}^{d}\right)$ наличие L-предела эквивалентно условию (1).

Доказательство. Мера

$$
\mu\left(T_{i} A \cap S_{i} A \cap A\right)
$$

стремится к нулю, следовательно, согласно утверждению 3 она равна 0 с некоторого момента. Осталось заметить, что из равенства

$$
\mu\left(T_{i} A \cap S_{i} A \cap A\right)=0
$$

следует равенство

$$
\mu\left(T_{i}^{2^{k}} A \cap S_{i}^{2^{k}} A \cap A\right)=0
$$

для любого натурального $k$.

Еще одна вариация $L$-систем состоит в замене предельного отношения $(2)$ на соотношение

$$
\mu\left(T_{i} A \cap S_{i} A \cap A\right) \rightarrow \Delta .
$$

Такие пределы будем называть $L_{\Delta}$-пределами.

СледствиЕ 8. При любом достаточно малом $\Delta$ перемешивающий поток (многомерный или одномерный) не обладает $L_{\Delta}$-пределом.

ДокАзАтельство. Анализ доказательства утверждения 2 показывает, что при достаточно малом $\Delta$ мера $\mu(U A \cap A)$ мало отличается от $1 / 4$ или $1 / 2$. Однако для любого множества $A$ меры $1 / 2$ существует преобразование $U$, коммутирующее с многомерным потоком и такое, что

противоречие.

$$
\mu(U A \cap A)=\frac{3}{8},
$$

Результаты данной работы позволяют также исследовать асимметрию в $L$-системах в смысле работ [10] и [11].

Автор выражает благодарность А. М. Стёпину, Ж.-П. Тувено и К. Шмидту и особенно В. В. Рыжикову за полезные обсуждения работы. 


\section{СПИСОК ЦИТИРОВАННОЙ ЛИТЕРАТУРЫ}

[1] В. А. Рохлин, Изв. АН СССР. Сер. матем., 13:4 (1949), 329-340. [2] В. В. Рыжиков, Матем. сб., 183:3 (1992), 133-160. [3] F. Ledrappier, C. R. Acad. Sci. Paris Sèr. A-B, 287:7 (1978), А561-A563. [4] С. В. Тихонов, Матем. заметки, 95:2 (2014), 282-299. [5] С.В. Тихонов, УМН, 67:4 (2012), 187-188. [6] В.В. Рыжиков, УМH, 44:1 (1989), 205-206. [7] B. Host, Israel J. Math., 76:3 (1991), 289-298. [8] L. Arenas-Carmona, D. Berend, V. Bergelson, Ergodic Theory Dynam. Systems, 28:2 (2008), 339-365. [9] T. de la Rue, Bull. Braz. Math. Soc. (N.S.), 37:4 (2006), 503-521. [10] И. С. Ярославцев, Матем. заметки, 95:3 (2014), 479-480. [11] В. В. Рыжиков, Матем. заметки, 96:3 (2014), 432-439.

С. В. Тихонов

Российский экономический университет им. Г. В. Плеханова, г. Москва

E-mail: tikhonovc@mail.ru
Поступило

30.07 .2014

Исправленный вариант

03.10 .2014 